\title{
Maximisation of the yield of final product on substrate in the case of sequential reactions catalysed by coimmobilised enzymes: a theoretical analysis
}

\author{
Jesus F. Celayeta, André H. Silva, Victor M. Balcão, F. Xavier Malcata
}

\begin{abstract}
A theoretical procedure is reported, which aims $C_{E_{i}}$ at finding the best molar distribution of two enzymes $C_{E_{i}}^{*}$ (considered equivalent and mutually exclusive in terms of $C_{E, \text { tot }}$ occupancy of immobilisation sites), coimmobilised in a slab-shaped porous bead, that catalyse two sequential irreversible reactions (involving an initial reactant species, $D_{\mathrm{ap}, i}$ an intermediate species and a final product species, at 1:1:1 stoichiometry) following Michaelis-Menten kinetics. The mathematical derivation uses as objective function maximisation of yield of the final product on the initial reactant $K_{m, i}$ coupled with the maximum catalyst effectiveness. The Thiele modulus was found to drop out as a relevant parameter for the determination of the optimum concentration of the intermediate (reactant) species, although it plays a role in the best distribution of enzyme within the slab. Low values of $k_{\text {cat }}$ for the first enzyme and high values of surface concentration of the initial reactant lead to small Thiele moduli; such diffusional regime promotes retention of product(s) within the porous matrix, thus providing opportunities for more complete conversion to the final product. The maximum yield of the final product corresponds to immobilising most of the first enzyme in the innermost portion of the slab and most of the second enzyme in the outermost portion.
\end{abstract}

\begin{tabular}{|c|c|}
\hline \multicolumn{2}{|c|}{ Abbreviations and symbols } \\
\hline$A_{i}$ & $\begin{array}{l}\text { reaction species ( } i=1 \text { (departing substrate), } \\
2 \text { (intermediate species), } 3 \text { (final product)) (-) }\end{array}$ \\
\hline$C_{A_{i}}$ & mole concentration of $A_{i}\left(\mathrm{~mol} \times \mathrm{m}^{-3}\right)$ \\
\hline$C_{A_{i}}^{*}$ & normalised counterpart of $C_{A_{i}}(-)$ \\
\hline$C_{A_{i}, 0}$ & $\begin{array}{l}\text { mole concentration of } A_{i} \text { on the surface of the } \\
\text { slab }\left(\mathrm{mol} \times \mathrm{m}^{-3}\right)\end{array}$ \\
\hline$C_{A_{i}, 0}^{*}$ & normalised counterpart of $C_{A_{i}, 0}(-)$ \\
\hline
\end{tabular}

J.F. Celayeta, A.H. Silva, V.M. Balcão, F.X. Malcata ( $\square)$

Escola Superior de Biotecnologia,

Universidade Católica Portuguesa,

Rua Dr. António Bernardino de Almeida, 4200-072 Porto, Portugal

\section{J.F. Celayeta}

Department of Process Engineering,

University College, Cork, Ireland

\section{V.M. Balcão}

Universidade Fernando Pessoa,

Praça 9 de Abril, 4249-004 Porto, Portugal mole concentration of enzyme $E_{i}\left(\mathrm{~mol} \times \mathrm{m}^{-3}\right)$ normalised counterpart of $C_{E_{i}}(-)$

maximum number of moles of enzyme that can be immobilised per unit volume of catalyst $\left(\mathrm{mol} \times \mathrm{m}^{-3}\right)$

apparent diffusivity of $A_{i}$ in the bead porous matrix $(i=1,2,3)\left(\mathrm{m}^{2} \times \mathrm{s}^{-1}\right)$

$k_{\text {cat }, \mathrm{i}}$ pseudo-first-order catalytic constant associated with the $i$-th enzyme $(i=1,2)\left(\mathrm{s}^{-1}\right)$

Michaelis-Menten constant associated with the $i$-th enzyme $(i=1,2)\left({\left.\mathrm{mol} \times \mathrm{m}^{-3}\right)}^{-}\right.$

$K_{m, i}^{*} \quad$ dimensionless counterpart of $K_{\mathrm{m}, \mathrm{i}}(-)$

$L^{m, i} \quad$ half-thickness of the slab (m)

$p_{1} \quad$ auxiliary variable (-)

$T h_{1} \quad$ Thiele modulus associated with $A_{1}$ and $E_{1}(-)$

$x \quad$ longitudinal coordinate of the slab $(\mathrm{m})$

$x^{\star} \quad$ normalised counterpart of $x(-)$

$Y_{A_{3}, A_{1}} \quad$ yield of $A_{3}$ on $A_{1}(-)$

$\alpha \quad$ integration constant (-)

$\omega_{i} \quad$ ratio of diffusivities of substrates $A_{i}$ and $A_{1}(i=2$,

3) $(-)$

$\begin{array}{ll}\eta_{2} & \text { ratio of } k_{\text {cat }, 2} \text { and } k_{\text {cat, } 1}(-) \\ \Phi_{2} & \text { auxiliary variable (-) }\end{array}$

$\Omega \quad$ auxiliary function (-)

\section{Introduction}

Enzymes are proteins that possess catalytic properties arising from their capacity for specific activation (Whitaker 1972). The intrinsic three-dimensional architecture of such (ubiquitous) biological catalysts makes them particularly active and selective, so practical advantage has for decades been taken from these proteins in terms of industrial processing. Examples of processes based on (immobilised) enzymes which have met with commercial success include (but are not limited to) use of aminoacylase for synthesis of enantiomerically pure L-amino acids (Chibata et al. 1972), use of glucose isomerase for production of high fructose syrups from glucose aqueous solutions (Venkatasubramanian and Harrow 1979), and use of lipase for controlled hydrolysis of butterfat (Malcata and Hill 1995) or interesterification of butterfat with oleic acid (Balcão and Malcata 1998, Balcão et al. 1998). There are three major reasons why immobilised enzymes (i.e. enzymes retained via containment by, or attachment to a condensed phase that is independent of the fluid reaction phase and is easily separated from it via bulk mechanical forces) are desirable: (i) retention of enzyme in the bead (and hence in the reactor) avoids need for upstream enzyme makeup to the reactor and prevents downstream contamination of the product by 
residual enzyme; (ii) immobilised enzymes retain their activity longer than those in solution [because the natural flexibility of the proteinaceous backbone, essential for thermal denaturation to occur, is restricted to some extent by immobilisation, although other forms of deactivation obviously exist (Balcão et al. 2001a, Mozhaev and Martinek 1982)]; and (iii) immobilised enzymes may be deliberately fixed in position near other enzymes participating in a reaction sequence, thereby increasing the catalytic efficiency of multistep conversions [because the entropic contribution arising from the requirement of productive collision of the product of the first reaction with the enzyme catalysing the second reaction is dramatically reduced when the interparticle, completely random phenomenon, becomes an intraparticle, more ordered phenomenon (Mosbach and Mattiasson 1976, Bailey and Ollis 1986, Balcão et al. 2001b)].

Examples of processes based on coimmobilised enzymes include (but are not limited to): (i) the use of coimmobilised lactate-dehydrogenase and lactate-

monooxygenase before an oxygen electrode to produce a biosensor having identical sensitivity for lactic and pyruvic acids, or coimmobilised laccase and glucose-oxidase to eliminate ascorbic acid, uric acid or bilirubin during glucose analysis (Wollenberger et al. 1987); (ii) the use of coimmobilised lactate-dehydrogenase, lactate-oxidase and catalase in the column of an enzyme thermistor for the determination of L-lactate, and of a similar system employing coimmobilised glucose-oxidase, catalase and glucose-dehydrogenase (Scheller et al. 1985); (iii) the coimmobilisation of D-amino acid oxidase and catalase for the quantitative transformation of D-phenylalanine into phenylpyruvic acid (Fernández-Lafuente et al. 1998); (iv) the coimmobilisation of glucoamylase and glucose isomerase for the one-step conversion of dextrin to fructose ( $\mathrm{Ge}$ et al. 1999); and (v) the coimmobilisation of L-asparaginase and glutamate dehydrogenase for the extracorporeal treatment of leukaemia (Balcão et al. 2001b).

Considerations on yields and selectivities of sequential reactions have been described thoroughly in the chemical engineering literature (e.g. Hill 1977, Smith 1981). In the case of immobilised catalysts, it is well accepted that the intermediate product molecule formed within the porous catalyst structure (say, $A_{2}$ ) from the initial reactant (say, $A_{1}$ ) has a high probability of reacting further (to become, say, $A_{3}$ ) before it can diffuse out of the bead (Hill 1977, Fernández-Lafuente et al. 1998, Riley et al. 1999). Hence, sequential reactions should to advantage be brought about by immobilised catalysts, under operating conditions where diffusional resistance plays a role, if the yield of final product on initial reactant (i.e. $\left(C_{A_{3}}-C_{A_{3,0}}\right) /\left(C_{A_{1,0}}-C_{A_{1}}\right)$, where subscript 0 denotes initial, or inlet conditions) is to be maintained as high as possible; however, a compromise should always be reached because, due to diffusional limitations imposed on the overall process, small values for the Thiele modulus also promote low overall conversion (i.e. $\left.\left(C_{A_{1,0}}-C_{A_{1}}\right) / C_{A_{1,0}}\right)$ for a given reaction time, or reactor space time. The design and simulation of pellets with various shapes containing immobilised single enzymes for various rate equations have been considered by Malcata (1987); later, the same author (Malcata 1991) provided an analytical solution for the substrate concentration profile which optimises a given economic criterion. The problem of improving the effectiveness factor of an immobilised enzyme via bulk convection through a catalyst slab has been considered by Moreira and Malcata (1996), whereas the advantages and disadvantages associated with use of immobilised enzymes in what concerns enhancement of product selectivity for sequential degradation reactions of oligomeric substrates have been tackled by Varga and Malcata (1996).

Despite the aforementioned contributions, apparently no effort has to date been devoted to the problem of multiple coimmobilised enzymes. Therefore, it is the purpose of this communication to report on a theoretical procedure aimed at finding the best molar distribution of two enzymes (considered equivalent and mutually exclusive, in terms of occupancy of immobilisation sites), coimmobilised in a slab-shaped porous bead, that catalyse two sequential irreversible reactions involving the substrate, an intermediate compound and the final product, with 1:1:1 stoichiometry as described by the classical Michaelis-Menten mechanism. The mathematical derivation will depart from imposition of the necessary condition associated with the maximum yield (ratio of concentration of final product relative to initial reactant) and the maximum catalyst effectiveness, and will allow conclusions to be drawn from a rather limited number of dimensionless parameters.

\section{Theory}

Assume that the following set of two sequential irreversible reactions is under consideration:

$A_{1} \underset{K_{m, 1}}{\stackrel{k_{\text {cat }, 1}}{\longrightarrow}} A_{2} \underset{K_{m, 2}}{\stackrel{k_{\text {cat }, 2}}{\longrightarrow}} A_{3}$

where $A_{1}$ and $A_{2}$ denote the substrates for the first and the second enzyme, respectively, and $A_{3}$ represents the final product, $k_{\mathrm{cat}, i}$ a pseudo-first-order catalytic constant for the $i$-th enzyme, and $K_{m, i}$ the Michaelis-Menten parameter for the $i$-th enzyme. If the two enzymes involved in the aforementioned catalytic cascade are coimmobilised in a slab-shaped porous bead, and if the immobilisation of either enzyme requires the same number (and layout) of surface sites for adsorption or covalent attachment, then the mass balances to the initial substrate, the intermediate species and the final product read

$$
\begin{aligned}
& -\mathrm{D}_{\mathrm{ap}, 1} \frac{d^{2} C_{A_{1}}}{d x^{2}}+\frac{k_{\mathrm{cat}, 1} C_{E_{1}} C_{A_{1}}}{K_{m, 1}+C_{A_{1}}}=0 \\
& -\mathrm{D}_{\mathrm{ap}, 2} \frac{d^{2} C_{A_{2}}}{d x^{2}}-\frac{k_{\mathrm{cat}, 1} C_{E_{1}} C_{A_{1}}}{K_{m, 1}+C_{A_{1}}}+\frac{k_{\mathrm{cat}, 2} C_{E_{2}} C_{A_{2}}}{K_{m, 2}+C_{A_{2}}}=0 \\
& -\mathrm{D}_{\mathrm{ap}, 3} \frac{d^{2} C_{A_{3}}}{d x^{2}}-\frac{k_{\mathrm{cat}, 2} C_{E_{2}} C_{A_{2}}}{K_{m, 2}+C_{A_{2}}}=0 \\
& x=0, \quad \frac{d C_{A_{1}}}{d x}=\frac{d C_{A_{2}}}{d x}=\frac{d C_{A_{3}}}{d x}=0 \\
& x=L, C_{A_{1}}=C_{A_{1}, 0}, C_{A_{2}}=C_{A_{2}, 0}, C_{A_{3}}=C_{A_{3}, 0}
\end{aligned}
$$

where $D_{\mathrm{ap}, i}$ denotes the apparent diffusivity of compound $A_{i}$ in the bead porous matrix, $C_{A_{i}}$ the molar concentration 
of substrate $A_{i}, C_{A_{i}, 0}$ the molar concentration of compound $A_{i}$ on the surface of the slab, $C_{E_{i}}$ the molar concentration of enzyme $E_{i}, x$ the longitudinal coordinate along the slab, and $L$ the half-thickness of the slab. Denoting by $C_{E \text {,tot }}$ the maximum number of moles of (either) enzyme that can be immobilised per unit volume of catalyst, and algebraically rearranging Eq. 2, one obtains

$\frac{d^{2} C_{A_{1}}^{*}}{d x^{*} 2}-\frac{T h_{1}^{2} C_{E_{1}}^{*} C_{A_{1}}^{*}}{K_{m, 1}^{*}+C_{A_{1}}^{*}}=0$

$\frac{d^{2} C_{A_{2}}^{*}}{d x^{*} 2}-\frac{T h_{1}^{2} C_{E_{1}}^{*} C_{A_{1}}^{*}}{\omega_{2}\left(K_{m, 1}^{*}+C_{A_{1}}^{*}\right)}+\frac{T h_{1}^{2} \eta_{2}\left(1-C_{E_{1}}^{*}\right) C_{A_{2}}^{*}}{\omega_{2}\left(K_{m, 2}^{*}+C_{A_{2}}^{*}\right)}=0$

$\frac{d^{2} C_{A_{3}}^{*}}{d x^{*} 2}+\frac{T h_{1}^{2} \eta_{2}\left(1-C_{E_{1}}^{*}\right) C_{A_{2}}^{*}}{\omega_{2}\left(K_{m, 2}^{*}+C_{A_{2}}^{*}\right)}=0$

$x^{*}=0, \quad \frac{d C_{A_{1}}^{*}}{d x}=\frac{d C_{A_{2}}^{*}}{d x}=\frac{d C_{A_{3}}^{*}}{d x}=0$

$x^{*}=1, \quad C_{A_{1}}^{*}=1, C_{A_{2}}^{*}=C_{A_{2}, 0}^{*}, C_{A_{3}}^{*}=C_{A_{3}, 0}^{*}$

where the normalised variables are defined by

$C_{A_{i}}^{*} \equiv \frac{C_{A_{i}}}{C_{A_{1}, 0}}(i=1,2,3)$

$x^{*} \equiv \frac{x}{L}$

$C_{E_{1}}^{*} \equiv \frac{C_{E_{1}}}{C_{E, \text { tot }}}$

$C_{A_{i}, 0}^{*} \equiv \frac{C_{A_{i}, 0}}{C_{A_{1}, 0}}(i=1,2,3)$

and where the dimensionless parameters are defined as

$T h_{1} \equiv L \sqrt{\frac{k_{\mathrm{cat}, 1} C_{E, \mathrm{tot}}}{C_{A_{1}, 0} \mathrm{D}_{a p, 1}}}$

$K_{m, i}^{*} \equiv \frac{K_{m, i}}{C_{A_{1}, 0}} \quad(i=1,2,3)$

$\omega_{i} \equiv \frac{\mathrm{D}_{a p, i}}{\mathrm{D}_{a p, 1}}(i=2,3)$

$\eta_{2} \equiv \frac{k_{\text {cat }, 2}}{k_{\text {cat }, 1}}$

The yield of product $A_{3}$ on reagent $A_{1}$, defined as

$Y_{A_{3}, A_{1}}=-\frac{\mathrm{D}_{a p, 3}\left(\frac{d C_{A_{3}}}{d x}\right)_{x=L}}{\mathrm{D}_{a p, 1}\left(\frac{d C_{A_{1}}}{d x}\right)_{x=L}}=-\omega_{3} \frac{\left(\frac{d C_{A_{3}}^{*}}{d x^{*}}\right)_{x^{*}=1}}{\left(\frac{d C_{A_{1}}^{*}}{d x^{*}}\right)_{x^{*}=1}}$

will then be maximum if, following generation, substrate $A_{2}$ will eventually not leave the porous slab as such (because, being immediately available to the second enzyme, it is instantly degraded). Since the compounds are related by 1:1:1 stoichiometry and are assumed to be transported by molecular diffusion only, then this assertion is mathematically equivalent to stating that

$Y_{A_{3}, A_{1}}=1$
On the other hand, if a gradient of $C_{A_{2}}$ existed, then some molecules of substrate $A_{2}$ would be, upon generation by the first chemical reaction depicted in Eq. 1, transported by molecular diffusion prior to depletion via the second reaction depicted in Eq. 2; in such a situation, the diffusional resistance associated with the tortuosity of the pores and the porosity of the bead should, for modelling purposes, be added to the intrinsic kinetic resistance of the chemical reaction itself (which would thus result in a slower overall process than if no gradient of $A_{2}$ built up). Therefore, the absence of a concentration gradient in $A_{2}$ will permit the highest possible effectiveness for the catalyst slab, and hence Eq. 7i may be replaced by

$\left(\frac{d C_{A_{2}}^{*}}{d x^{*}}\right)_{x^{*}=1}=0$

The aforementioned reasoning, coupled with the simple stoichiometry of the reaction sequence as apparent in Eq. 3, degenerates to a set of one ordinary differential equation and two algebraic equations, viz.

$\frac{d^{2} C_{A_{1}}^{*}}{d x^{*} 2}-\frac{T h_{1}^{2} C_{E_{1}}^{*} C_{A_{1}}^{*}}{K_{m, 1}^{*}+C_{A_{1}}^{*}}=0$

$\frac{C_{E_{1}}^{*} C_{A_{1}}^{*}}{K_{m, 1}^{*}+C_{A_{1}}^{*}}-\frac{\eta_{2}\left(1-C_{E_{1}}^{*}\right) C_{A_{2}, 0}^{*}}{K_{m, 2}^{*}+C_{A_{2}, 0}^{*}}=0$

$\omega_{3}\left(C_{A_{3}}^{*}-C_{A_{3}, 0}^{*}\right)=1-C_{A_{1}}^{*}$

$x^{*}=0, \quad \frac{d C_{A_{1}}^{*}}{d x^{*}}=0$

$x^{*}=1, \quad C_{A_{1}}^{*}=1$

Combination of the first two equalities in Eq. 8 leads to $\frac{d^{2} C_{A_{1}}^{*}}{d x^{*} 2}=\frac{T h_{1}^{2} \Phi_{2} C_{A_{1}}^{*}}{C_{A_{1}}^{*}+K_{m, 1}^{*} \Phi_{2}}$

$C_{E_{1}}^{*}=\frac{\Phi_{2}\left(K_{m, 1}^{*}+C_{A_{1}}^{*}\right)}{C_{A_{1}}^{*}+K_{m, 1}^{*} \Phi_{2}}$

which allows sequential solution with respect to $C_{A_{1}}^{*}\left\{x^{*}\right\}$ and $C_{E_{1}}^{*}\left\{x^{*}\right\}$ for a given $\Phi_{2}$, knowing that the auxiliary variable $\Phi_{2}$ is defined as

$\Phi_{2} \equiv \frac{\eta_{2} C_{A_{2}, 0}^{*}}{K_{m, 2}^{*}+\left(1+\eta_{2}\right) C_{A_{2}, 0}^{*}}$

The first equality in Eq. 9 may be transformed by a suitable variable change (Stephenson 1973) into

$p_{1} \frac{d p_{1}}{d C_{A_{1}}^{*}}=\frac{T h_{1}^{2} \Phi_{2} C_{A_{1}}^{*}}{C_{A_{1}}^{*}+K_{m, 1}^{*} \Phi_{2}}$

where the auxiliary variable $p_{1}$ is defined as

$p_{1} \equiv \frac{d C_{A_{1}}^{*}}{d x^{*}}$ 
Integration of Eq. 11 gives

$$
\frac{1}{2 T h_{1}^{2}} p_{1}^{2}=\Phi_{2} C_{A_{1}}^{*}-K_{m, 1}^{*} \Phi_{2}^{2} \ln \left\{C_{A_{1}}^{*}+K_{m, 1}^{*} \Phi_{2}\right\}+\alpha
$$

where $\alpha$ denotes an arbitrary integration constant. The symmetry boundary condition in Eq. 8 may be replaced by an alternative boundary condition, viz.

$C_{A_{1}}^{*}\left\{x^{*}=0\right\}=C_{A_{1} \text {,min }}^{*}$; this new boundary condition may be rewritten in a more convenient form as

$p_{1}\left\{C_{A_{i}}^{*}=C_{A_{1}, \min }^{*}\right\}=0$, which, upon combination with

Eq. 13, allows the arbitrary constant $\alpha$ to be defined by

$\alpha \equiv-\Phi_{2} C_{A_{1}, \min }^{*}+K_{m, 1}^{*} \Phi_{2}^{2} \ln \left\{C_{A_{1}, \min }^{*}+K_{m, 1}^{*} \Phi_{2}\right\}$

The value of the surface concentration of substrate $A_{2}$ (i.e. $C_{A_{2}, 0}^{*}$ ) must be known a priori as a boundary condition, in order for the ordinary differential equation denoted as Eq. 2 be fully specified; however, as of now its value has not yet been fixed. A reasonable choice would be the value that leads to the steepest gradient for the concentration of $A_{1}$ in the immediate vicinity of the surface of the slab (or, equivalently, to the highest effectiveness factor with respect to consumption of substrate $\left.A_{1}\right)$. Mathematically, such a choice corresponds to

$\frac{d\left(p_{1}\right)_{C_{A_{1}}^{*}=1}}{d C_{A_{2}, 0}^{*}}=0$

which, after combination with Eqs. 13 and 14 and further simplification, becomes

$$
\begin{gathered}
1-C_{A_{1}, \min }^{*}-K_{m, 1}^{*} 2 \Phi_{2}^{2} \frac{C_{A_{1}, \min }^{*}-1}{\left(1+K_{m, 1}^{*} \Phi_{2}\right)\left(C_{A_{1}, \min }^{*}+K_{m, 1}^{*} \Phi_{2}\right)} \\
-\cdots 2 K_{m, 1}^{*} \Phi_{2} \ln \left\{\frac{1+K_{m, 1}^{*} \Phi_{2}}{C_{A_{1}, \min }^{*}+K_{m, 1}^{*} \Phi_{2}}\right\}=0
\end{gathered}
$$

where advantage was taken from the fact that both $p_{1}$ and $d \Phi / d C_{A_{2}, 0}^{*}$ take positive finite values irrespective of $C_{A_{2}, 0}^{*}$. The value sought for $C_{A_{2}, 0}^{*}$ may now be found by numerical solution of Eq. 16 with respect to $\Phi_{2}$, and subsequent solution of Eq. 10 with respect to $C_{A_{2}, 0}^{*}$ using the appropriate values of $\eta_{2}$ and $K_{m, 2}^{*}$. A plot of $\Phi_{2}$ as a simultaneous function of $C_{A_{1}, \text { min }}^{*}$ and $K_{m, 1}^{*}$ is depicted in Fig. 1.

The result for $\Phi_{2}=\Phi_{2}\left\{K_{m, 1}^{*}, C_{A_{1}, \min }^{*}\right\}$, as conveyed by Eq. 16, may now be used in Eq. 13, which, upon integration, yields the alternative form

$$
\begin{aligned}
& \sqrt{2 \Phi_{2}} T h_{1} x^{*} \\
& =\cdots \int_{C_{A_{1}, \min }^{*}}^{C_{A_{1}}^{*}} \frac{d C_{A_{1}}^{*}}{\sqrt{C_{A_{1}}^{*}-C_{A_{1}, \min }^{*}-K_{m, 1}^{*} \Phi_{2} \ln \left\{\frac{C_{A_{1}}^{*}+K_{m, 1}^{*} \Phi_{2}}{C_{A_{1}, \min }^{*}+K_{m, 1}^{*} \Phi_{2}}\right\}}}
\end{aligned}
$$

for the computation of the concentration profile of $C_{A_{1}}^{*}$, where $C_{A_{1}, \min }^{*}$ is obtained via numerical solution of

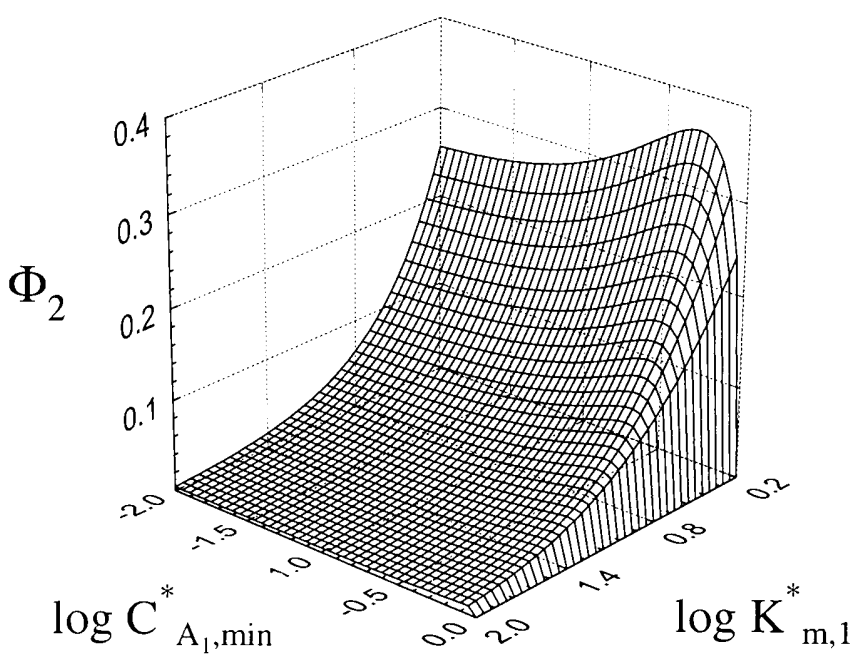

Fig. 1. Three-dimensional plot of $\Phi_{2}$ versus $\log \left(K_{m, 1}^{*}\right)$ and $\log \left(C_{A_{1}, \text { min }}^{*}\right)$

$$
=\cdots \int_{C_{A_{1}, \min }^{*}}^{\sqrt{2 \Phi_{2}} T h_{1}} \frac{d C_{A_{1}}^{*}}{\sqrt{C_{A_{1}}^{*}-C_{A_{1}, \min }^{*}-K_{m, 1}^{*} \Phi_{2} \ln \frac{\left\{C_{A_{1}}^{*}+K_{m, 1}^{*} \Phi_{2}\right\}}{C_{A_{1}, \min }^{*}+K_{m, 1}^{*} \Phi_{2}}}}
$$

Numerical solution of either Eq. 17 or Eq. 18 is somewhat difficult because there is a singularity at the lower limit of integration. For this reason, it is convenient to apply the rule of integration by parts to both equations in order to remove such an ill-behaved point; the results of such process are

$$
\begin{aligned}
& \sqrt{2 \Phi_{2}} T h_{1} x^{*} \\
& =\cdots \frac{2 \sqrt{C_{A_{1}}^{*}-C_{A_{1}, \min }^{*}-K_{m, 1}^{*} \Phi_{2} \ln \frac{\left\{C_{A_{1}}^{*}+K_{m, 1}^{*} \Phi_{2}\right\}}{C_{A_{1}, \min }^{*}+K_{m, 1}^{*} \Phi_{2}}}}{1-\frac{K_{m, 1}^{*} \Phi_{2}}{C_{A_{1}}^{*}+K_{m, 1}^{*} \Phi_{2}}} \\
& +\cdots 2 \int_{C_{A_{1}, \min }^{*}}^{C_{A_{1}}^{*}} \frac{K_{m, 1}^{*} \Phi_{2} \sqrt{C_{A_{1}}^{*}-C_{A_{1}, \min }^{*}-K_{m, 1}^{*} \Phi_{2} \ln \frac{\left\{C_{A_{1}}^{*}+K_{m, 1}^{*} \Phi_{2}\right\}}{C_{A_{1}, \min }^{*}+K_{m, 1}^{*} \Phi_{2}}}}{C_{A_{1}}^{*} 2} d C_{A_{1}}^{*}
\end{aligned}
$$

and

$$
\begin{aligned}
& \sqrt{2 \Phi_{2}} T h_{1} \\
& =\cdots \frac{2 \sqrt{C_{A_{1}}^{*}-C_{A_{1}, \min }^{*}-K_{m, 1}^{*} \Phi_{2} \ln \frac{\left\{C_{A_{1}}^{*}+K_{m, 1}^{*} \Phi_{2}\right\}}{C_{A_{1}, \min }^{*}+K_{m, 1}^{*} \Phi_{2}}}}{1-\frac{K_{m, 1}^{*} \Phi_{2}}{C_{A_{1}}^{*}+K_{m, 1}^{*} \Phi_{2}}} \\
& +\cdots 2 \int_{C_{A_{1}, \min }^{*}}^{1} \frac{K_{m, 1}^{*} \Phi_{2} \sqrt{C_{A_{1}}^{*}-C_{A_{1}, \min }^{*}-K_{m, 1}^{*} \Phi_{2} \ln \frac{\left\{C_{A_{1}}^{*}+K_{m, 1}^{*} \Phi_{2}\right\}}{C_{A_{1}, \min }^{*}+K_{m, 1}^{*} \Phi_{2}}}}{C_{A_{1}}^{*} 2} d C_{A_{1}}^{*}
\end{aligned}
$$


respectively. Since, as apparent from Eq. 15,

$\Phi_{2}=\Phi_{2}\left\{K_{m, 1}^{*}, C_{A_{1}, \min }^{*}\right\}$, then Eq. 20 may be seen as a relation of the form $\Omega\left\{K_{m, 1}^{*}, C_{A_{1}, \min }^{*}\right\}=0$ (where $\Omega$ denotes a multiparametric function); this equation is thus to be numerically solved with respect to $C_{A_{1} \text {,min }}^{*}$ for each combination of values of $T h_{1}$ and $K_{m, 1}^{*}$. The graphical output of such a solution procedure is denoted as Fig. 2.

Once the concentration profile for $A_{1}$ has been calculated via Eq. 19, after combination with the adequate value of $C_{A_{1} \text {,min }}^{*}$, the best distribution of enzyme $E_{1}$ in the slab can be obtained from the second equality in Eq. 9, and the corresponding concentrations of $A_{3}$ and $A_{2}$ may be calculated from Eqs. 8 and 10. The profiles of $C_{A_{1}}^{*}$ for typical combinations of values of the relevant parameters $K_{m, 1}^{*}$, $T h_{1}$ and $\Phi_{2}$ are depicted in Fig. 3. The profiles of $C_{E_{1}}^{*}$ for the same combinations of values of the relevant parameters $K_{m, 1}^{*}, T h_{1}$ and $\Phi_{2}$ are depicted in Fig. 4.

\section{Discussion and conclusions}

It is interesting to note that $T h_{1}$ drops out as a relevant parameter for the determination of the optimum (constant) concentration of $A_{2}$, although it plays a role in the best distribution of enzyme within the slab. On the other hand, the lumped combination of parameters $\eta_{2}, K_{m, 2}^{*}$ and $C_{A_{2}, 0}^{*}$, given by $\Phi_{2}$ as defined in Eq. 10, rather than each of such parameters per se, contributes to the overall best distribution of enzyme.

A plot of $\Phi_{2}$ versus $K_{m, 1}^{*}$ or $C_{A_{1} \text {, min }}^{*}$ indicates that $\Phi_{2}$ takes physically realisable values only in the range $K_{m, 1}^{*}>10^{-1}$; such values do not violate the constraint $0<\Phi_{2}<1$, which is obtained as a result of coupling Eq. 16 with the fact that $K_{m, 2}^{*}, \eta_{2}$ and $C_{A_{2}, 0}^{*}$ take always positive values. Note that selection of said range for $K_{m, 1}^{*}$ will obviously not encompass all situations of practical interest, because often $K_{m, 1} \ll C_{A_{1}, 0}$; however, beyond that range no local optima can be found and the optimal solution of the problem will lie on a physical constraint, viz. $\Phi_{2}=1$ (as easily extrapolated from the trend depicted in Fig. 1). Inspection of Fig. 1 (which was constructed based on the aforementioned realisation) indicates that $\Phi_{2}$ increases monotonically when $K_{m, 1}^{*}$ decreases. However,

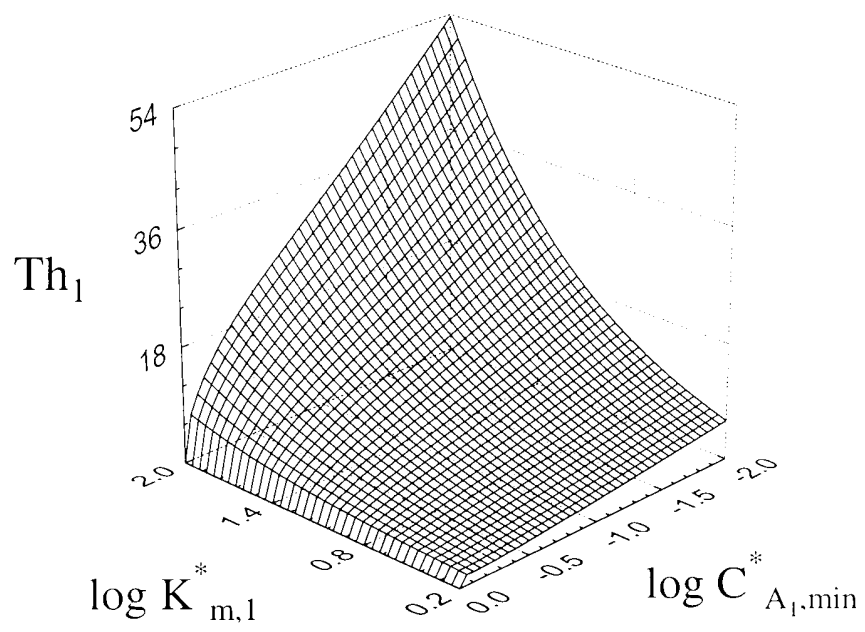

Fig. 2. Three-dimensional plot of $\log \left(C_{A_{1}, \text { min }}^{*}\right)$ versus $T h_{1}$ and $\log \left(K_{m, 1}^{*}\right)$
$\Phi_{2}$ goes through a maximum when $C_{A_{1}, \min }^{*}$ increases; this maximum occurs earlier for lower values of parameter $K_{m, 1}^{*}$. Note that higher values of $K_{m, 1}^{*}$ indicate a lower affinity of the enzyme for substrate $A_{1}$.
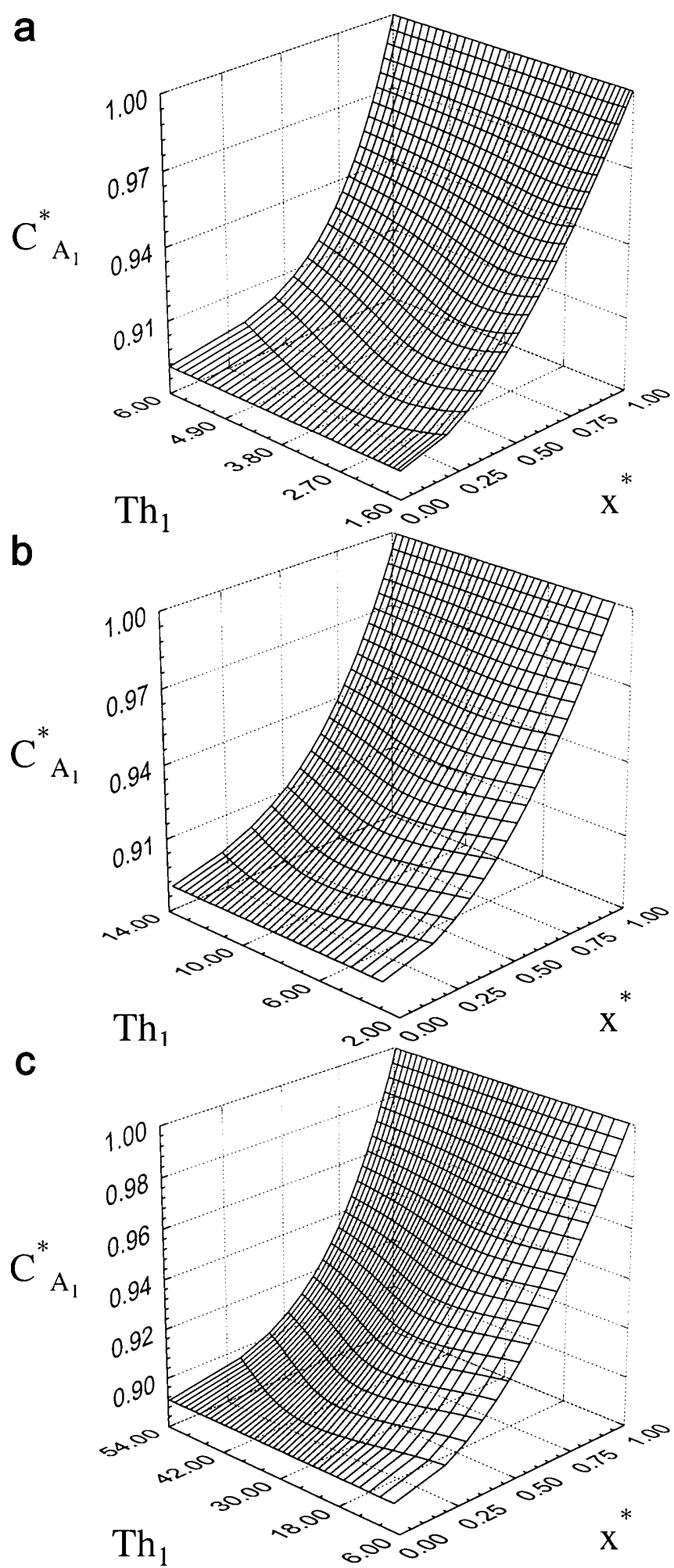

Fig. 3a-c. Three-dimensional plots of the concentration profile of $A_{1}$, viz. $C_{A_{1}}^{*}$, versus the longitudinal coordinate within the slab, viz. $x^{*}$, and the Thiele modulus, $T h_{1}$, associated with the maximum value for the yield of $A_{3}$ on $A_{1}$ and the maximum effectiveness factor, for $\log \left(K_{m, 1}^{*}\right)$ equal to a $0.2, \mathbf{b} 1$ and $\mathbf{c} 2$ 
Inspection of Fig. 2 indicates that, for higher values of $K_{m, 1}^{*}$ coupled with lower values of $C_{A_{1}, \text { min }}^{*}$, the values of $T h_{1}$
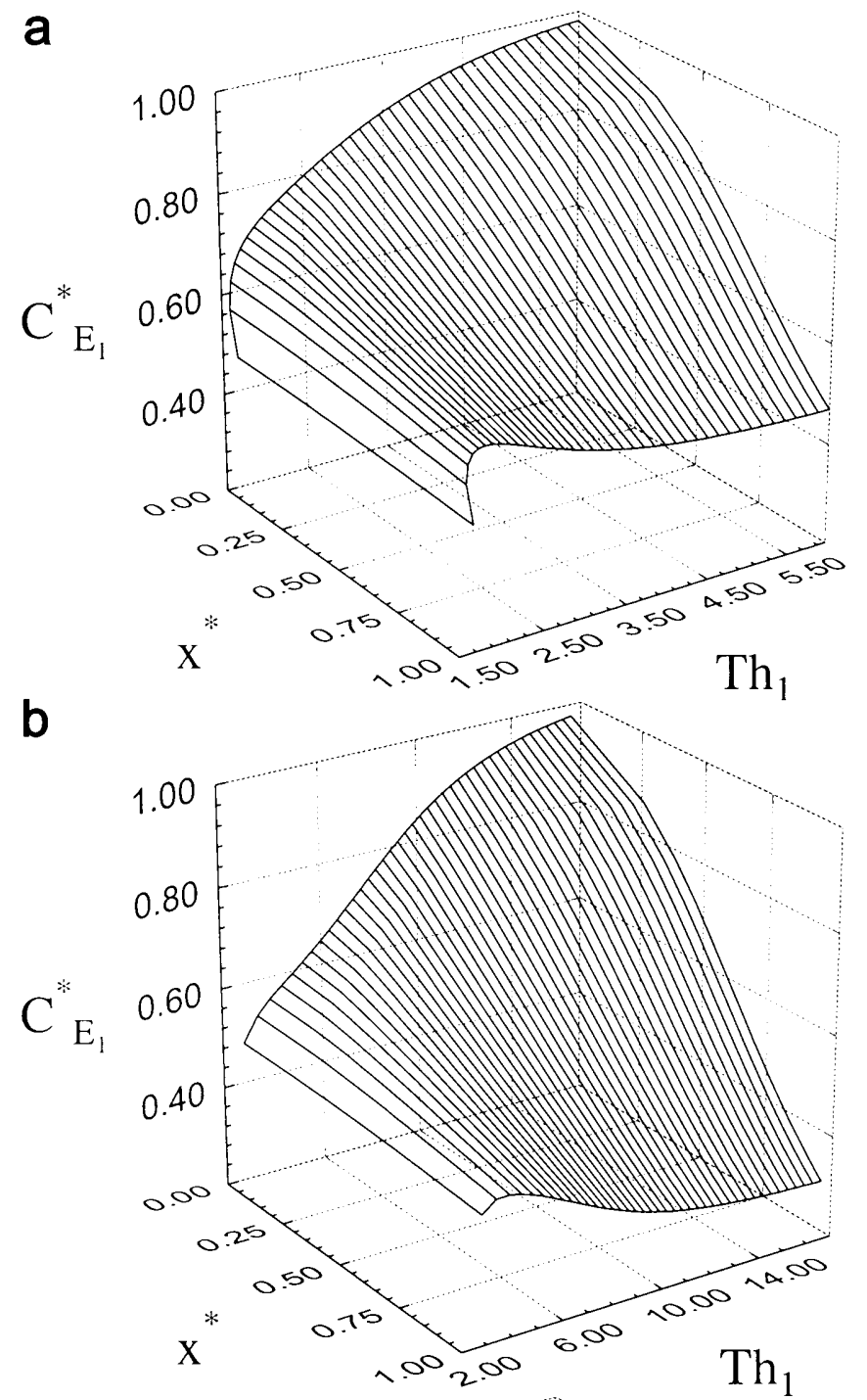

C

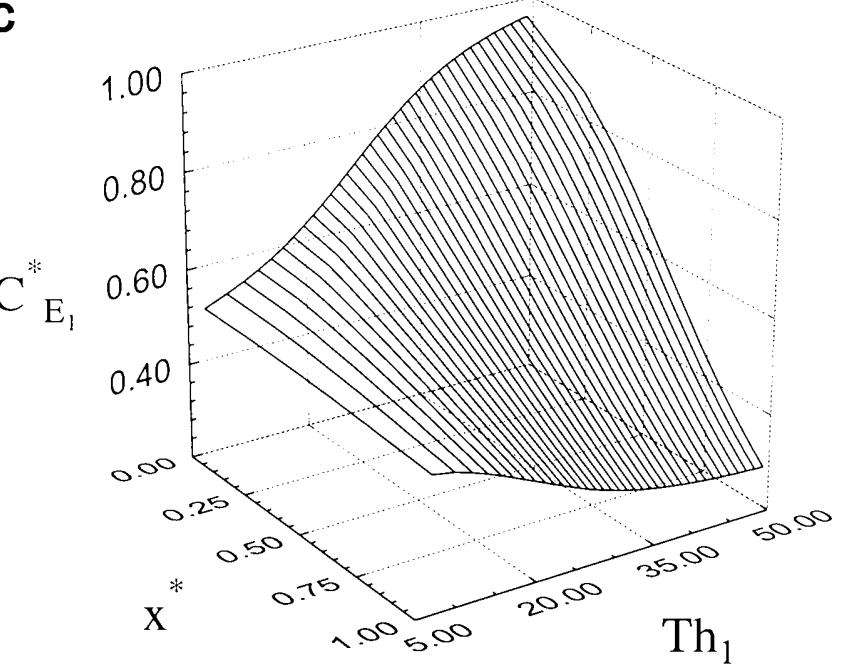

Fig. 4a-c. Three-dimensional plots of the concentration profile of $E_{1}$, viz. $C_{E_{1}}^{*}$, versus the longitudinal coordinate within the slab, viz. $x^{*}$, and the Thiele modulus, $T h_{1}$, associated with the maximum value for the yield of $A_{3}$ on $A_{1}$ and the maximum effectiveness factor, for $\log \left(K_{m, 1}^{*}\right)$ equal to a $0.2, \mathbf{b} 1$ and $\mathbf{c} 2$ become very high, which implies that the diffusional regime holds within the bead. This would promote retention of product(s) within the porous matrix, and hence provide opportunity for more complete conversion to the final product $A_{3}$. In general, $T h_{1}$ increases monotonically with both decrease in $C_{A_{1} \text {,min }}^{*}$ and increase in $K_{m, 1}^{*}$.

Inspection of Fig. 3 unfolds similar behaviour for all three orders of magnitude tested for $K_{m, 1}^{*}$. It also indicates that $C_{A_{i}}^{*}$ decreases as one gets more and more in depth into the slab, as expected; however, there is a value for $T h_{1}$ which, at each longitudinal location, yields a local minimum for $C_{A_{i}}^{*}$; interestingly, this effect vanishes as either the middle symmetry plane or the surface of the slab are approached. Those minima occur at higher values for parameter $T h_{1}$ when $K_{m, 1}^{*}$ increases.

Inspection of Fig. 4 indicates that the best fraction of $E_{1}$ immobilised within the porous slab at the symmetry plane decreases when $T h_{1}$ increases. In any case, the suggested trend for the maximum yield of $A_{3}$ on $A_{1}$ corresponds to immobilising most enzyme $E_{1}$ in the innermost portion of the slab, and most enzyme $E_{2}$ in the outermost portion. By doing so, most molecules of $A_{1}$ are forced to travel farther into the slab, where they are converted to product(s); since they are well within the matrix, they take longer to leave it, so there is plenty of opportunity for them to be further converted into $A_{3}$, which is eventually transported outward. This indication holds better, as anticipated, when diffusional resistance dominates; in fact, if the kinetic regime holds instead, no steep concentration gradients of products build up, so there is no need for a varying fraction of $E_{1}$ immobilised throughout $x^{\star}$. Finally, it should be noted that for lower values of $T h_{1}$, there should be an equimolar distribution of enzymes within the slab, whereas the decreasing fraction of $E_{1}$ immobilised as $x^{*}$ increases is convenient, in bioengineering practice, only if resistances to molecular transport play an important role.

The possibilities for practical application of the theoretical rationale developed above are multiple; one example pertains to the coimmobilisation of $\mathrm{L}$-asparaginase and glutamate dehydrogenase onto highly activated agaroseglutaraldehyde supports for the extracorporeal treatment of leukaemia (Balcão et al. 2001b). The sequential (irreversible) reaction in question involves the L-asparaginasemediated hydrolysis of L-asparagine $\left(A_{1}\right)$ with concomitant production of L-aspartic acid and ammonia $\left(A_{2}\right)$; the latter (intermediate) species is then taken up by the coimmobilised glutamate dehydrogenase in the presence of $\alpha$-ketoglutarate and $\beta$-NADH, to produce L-glutamic acid $\left(A_{3}\right)$ and $\mathrm{NAD}^{+}$. If degradation of L-asparagine were to be carried out solely by L-asparaginase, the ammonia produced would build up in the blood and eventually become a poison to the human kidney; such risk is easily overcome by coimmobilising glutamate dehydrogenase (which possesses a strong preference for ammonia), thus keeping the levels of the $A_{2}$ species low. However, owing to the high cost of such enzymes (as derived from extremely high purity requirements), their fractional and total amounts throughout the slab should be carefully determined, whereas total conversion of L-asparagine to L-glutamic acid should be sought (to prevent unnecessary extra effort by the kidneys). 


\section{References}

Bailey JE, Ollis DF (1986) Biochemical Engineering Fundamentals. McGraw-Hill, New York

Balcão VM, Malcata FX (1998) On the performance of a hollow-fiber bioreactor for acidolysis catalyzed by immobilized lipase. Biotechnol Bioeng 60:114-123

Balcão VM, Kemppinen A, Malcata FX, Kalo P (1998) Lipase-catalyzed acidolysis of butterfat with oleic acid: characterization of process and product. Enzyme Microb Technol 23:118-128

Balcão VM, Mateo C, Fernández-Lafuente R, Malcata FX, Guisán JM (2001a) Structural and functional stabilization of L-asparaginase via multi-subunit immobilization onto highly activated supports. Biotechnol Prog 17:537-542

Balcão VM, Mateo C, Fernández-Lafuente R, Malcata FX, Guisán JM (2001b) Coimmobilization of L-asparaginase and glutamate dehydrogenase onto highly activated supports. Enzyme Microb Technol 28:696-704

Chibata I, Tosa T, Sato T, Matsuo Y (1972) Preparation and industrial application of immobilised aminoacylases. In: Terui G (ed) Fermentation Technology Today. Society of Fermentation Technology, Japan, p 383

Fernández-Lafuente R, Rodriguez V, Guisán JM (1998) The coimmobilization of $\mathrm{D}$-amino acid oxidase and catalase enables the quantitative transformation of D-amino acids (D-phenylalanine) into $\alpha$-keto acids (phenylpyruvic acid). Enzyme Microb Technol 23:28-33

Ge Y, Wang Y, Zhou H, Tong Y, Li W (1999) Coimmobilization of glucoamylase and glucose isomerase by molecular deposition technique for one-step conversion of dextrin to fructose. J Biotechnol 67:33-40

Hill CG (1977) An Introduction to Chemical Engineering Kinetics and Reactor Design. Wiley, New York

Lamas EM, Barros RM, Balcão VM, Malcata FX (2001) Hydrolysis of whey proteins by proteases extracted from Cynara cardunculus and immobilized onto highly activated supports. Enz Microb Technol 28:642-662

Malcata FX (1987) Computer-aided design of pellets for fixed-bed reactors performing Michaëlis-Menten reactions. Comp Appl Biosci 3:297-301
Malcata FX (1991) On the analytical solution of a problem of optimisation in the field of immobilised enzymes. Int J Math Educ Sci Technol 22:177-185

Malcata FX, Hill CG (1995) Industrial utilisation of a hollow-fiber membrane reactor for the performance of controlled lipolysis of butterfat. Ann N Y Acad Sci 613:401-407

Moreira NE, Malcata FX (1996) Use of convective flow to enhance the effectiveness factor of porous slab-shaped beads with immobilised enzyme. J Chem Eng Jap 29:392-395

Mosbach K, Mattiasson B (1976) Multistep enzyme systems. Methods Enzymol 44:453-477

Mozhaev VV, Martinek K (1982) Inactivation and reactivation of proteins (enzymes). Enzyme Microb Technol 4:299-309

Riley MR, Muzzio FJ, Reyes SC (1999) Experimental and modelling studies of diffusion in immobilized cell systems. Appl Biochem Biotechnol 80:151-188

Scheller F, Siegbahn N, Danielsson B, Mosbach K (1985) Highsensitivity enzyme thermistor determination of L-lactate by substrate recycling using coimmobilized catalase, lactate-oxidase and lactate-dehydrogenase for enzyme electrode. Anal Chem 57:17401743

Smith JM (1981) Chemical Engineering Kinetics. McGraw-Hill International, Tokyo

Stephenson G (1973) Mathematical Methods for Science Students. Longman, London

Varga S, Malcata FX (1996) Enhancement of product selectivity via enzyme immobilisation in sequential degradation reactions of polymeric substrates. Bioprocess Eng 15:189-194

Venkatasubramanian K, Harrow HS (1979) Design of operation of a commercial immobilised glucose isomerase reactor system. Ann N Y Acad Sci 326:141-150

Whitaker JR (1972) Principles of Enzymology for the Food Sciences. Dekker, New York

Wollenberger U, Schubert F, Scheller F, Danielsson B, Mosbach K (1987) Coupled reactions with immobilized enzymes in biosensors. Bioelectrochem Biotechnol 119:167-170 